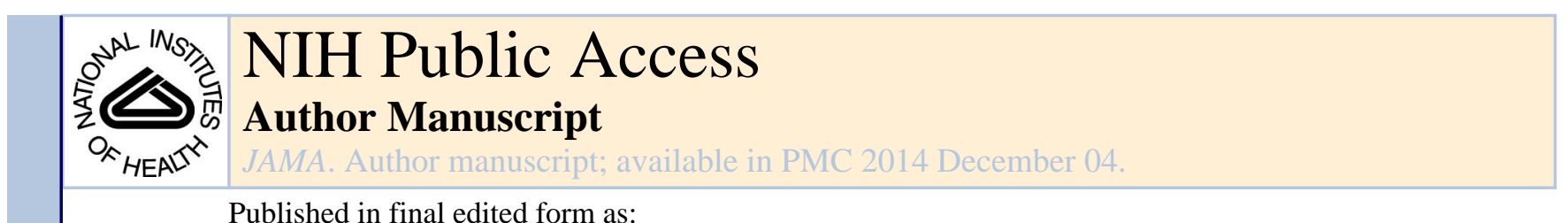

Published in final edited form as:

JAMA. 2012 June 6; 307(21): 2316-2317. doi:10.1001/jama.2012.5853.

\title{
Inhaled Hypertonic Saline in Infants and Young Children with Cystic Fibrosis
}

\author{
Elliott C. Dasenbrook, MD MHS ${ }^{1}$ and Michael W. Konstan, MD ${ }^{1}$ \\ ${ }^{1}$ Case Western Reserve University School of Medicine and Rainbow Babies and Children's \\ Hospital, Cleveland, $\mathrm{OH}$
}

Over the past 70 years, the survival of patients with cystic fibrosis (CF) has improved substantially[1]. However, median predicted survival is currently only 38 years of age[2].

Extending life expectancy to that of healthy individuals would presumably require initiating treatment in early infancy before the onset of clinically apparent disease in order to prevent irreversible lung damage. The recent advent of universal newborn screening for CF provides this opportunity.

Cystic fibrosis is caused by a mutation in the gene that encodes the cystic fibrosis transmembrane conductance regulator (CFTR) protein. Absence of functional CFTR at the surface of the airway epithelium in a CF patient results in decreased chloride secretion and increased sodium absorption, which causes depletion of water from the airway surface. This results in impaired mucociliary clearance, leading to a favorable environment for infection and inflammation to occur. Ultimately this leads to lung destruction[3]. Thus, restoring the salt and water balance in the CF airway has been the goal of a number of therapeutic strategies. In a recent clinical trial, ivacaftor, a potentiator of CFTR, was shown to improve lung function and decrease pulmonary exacerbations[4]. Although ivacaftor targets a specific mutation affecting only 4-5\% of CF patients worldwide, other CFTR specific investigational agents that have the potential to affect nearly all patients with $\mathrm{CF}$ are currently being studied. In the meantime, adding salt directly to the airway may be of benefit, and several studies have demonstrated the efficacy of this approach in patients with CF older than 6 years[5;6].

In this issue of JAMA, Rosenfeld and colleagues report results from a large, multicenter trial comparing inhaled $7 \%$ hypertonic saline to $0.9 \%$ saline (control) twice daily for 48 weeks in 321 infants and young children[7]. The study failed to achieve its primary endpoint, a reduction in the rate of pulmonary exacerbations during the 48 week treatment period. In addition, there were no significant differences between participants receiving hypertonic and isotonic saline for the secondary endpoints (height, weight, respiratory rate, oxygen saturation, cough and respiratory symptom score). Nonetheless, these trial results are important to the CF community, as the study identifies a treatment that should not be routinely used, demonstrates the ability to conduct a large, well-designed, international trial

Corresponding Author Michael W Konstan, MD, Rainbow Babies and Children's Hospital, 11100 Euclid Avenue, Cleveland, Ohio 44106, 216-844-3884 (O) 216-844-1479 (F), Michael.Konstan@uhhospitals.org. 
in very young patients with $\mathrm{CF}$, and raises the question of what endpoints are appropriate for evaluating a pulmonary therapy in relatively healthy infants and young children with CF.

The choice of rate of pulmonary exacerbation as the primary endpoint for this trial is relevant given that this outcome is universally recognized by clinicians as an important clinical endpoint that affects how a patient feels and functions and has been used in many $\mathrm{CF}$ clinical trials[8]. However, nearly all of the experience with the use of pulmonary exacerbation as an endpoint in CF clinical trials comes from patients greater than 6 years of age, in whom lung damage has already occurred and the goal has been to reverse existing pathology. In contrast, the participants in the study by Rosenfeld et al were age 4 months to 5 years and presumably had much less underlying lung disease to improve upon. In addition, although the authors used a protocol-defined definition for a pulmonary exacerbation, the type of exacerbations that occur in infants and young children likely differ from those in older children and adults with CF, primarily due to differences in the prevalence of viral infections and $P$. aeruginosa infections in these age groups. Powering the study on one of the secondary endpoints would have required many more participants to reach statistical significance in light of the modest differences between groups. As with exacerbation rates, there is no experience with the use of these secondary endpoints in interventional studies in infants and young children with CF. Change in pulmonary function measures is another often used endpoint in CF clinical trials in older children and adults and has been proposed for use in interventional studies of infants and young children[9].

Rosenfeld et al also performed infant pulmonary function tests as an exploratory endpoint in a subgroup of participants between 4 and 16 months of age. Examination of the 95\% confidence intervals of the differences in the adjusted mean change in the forced expiratory flows and volume between the hypertonic and isotonic saline groups suggests the possibility of some improvement with hypertonic saline. These exploratory results should be interpreted with the following considerations. Since more than a third of the participants did not have 2 pulmonary function measurements, bias could be introduced if participants with both measurements were clinically different (e.g. healthier and therefore able to undergo testing). This does not appear to be the case because the dropout rates between the hypertonic and isotonic saline groups were similar. Even if the primary endpoint of this study had been measures of infant pulmonary function and there was statistically significant improvement in $\mathrm{FEV}_{0.5}$ of $38 \mathrm{cc}$, how would a clinician interpret these results? A statistically significant difference does not necessarily imply clinical significance. While the infant pulmonary function results appear encouraging, given the limitations stated, these exploratory endpoints should be viewed as hypothesis generating and research exploring the clinical effecst of these differences is needed.

If traditional clinical endpoints are not sensitive enough to detect early, regional lung disease, then newer endpoints are required. An ideal endpoint would have the following characteristics: 1) sensitive to the progression of lung disease and the pathophysiology of $\mathrm{CF}, 2$ ) repeatable and reproducible, 3) quantifiable such that differences in magnitude between treatment groups will allow studies to be powered with a reasonable number of patients, 4) attainment of the measure is safe, minimally invasive, and does not require 
sedation, specialized equipment or technical expertise, and 5) available at nearly all CF centers for future use in clinical care. A daunting task!

Other potential endpoints that might meet most of these criteria include imaging and other modalities assessing lung function[9-11]. Chest computed tomography may be sensitive at detecting early lung disease due to its ability to detect regional disease. Computed tomography is limited for longitudinal studies, especially in infants, due to ionizing radiation. Magnetic resonance imaging is a potential alternative without the adverse effects of ionizing radiation and has been tested in patients with $\mathrm{CF}$ [12]. Lung clearance index is a measure of ventilation variability and, in a small, single-center study in CF patients with early stage lung disease appeared to improve with hypertonic saline[13]. In another study it was found to be sensitive and repeatable[14]. A primary challenge of these surrogate endpoints will be to link them to subsequent development or progression of lung disease. Future research is needed to determine which of these and other techniques, or combinations thereof, may be best suited to detect and follow early, regional CF lung disease[15], and will be useful as endpoints for clinical trials of therapeutic interventions in infants and young children with $\mathrm{CF}$.

How does the study by Rosenfeld et al assist clinicians and researchers? This study demonstrates that hypertonic saline offers no advantage over isotonic saline in decreasing exacerbations in infants and young children with CF. The current CF Foundation guidelines recommend the use of inhaled hypertonic saline only for patients age 6 years and older[16]. However, the final verdict on use of hypertonic saline for infants and young children has not been rendered. It would be disheartening if a viable therapeutic option was discarded because of negative study results when more sensitive endpoints may have detected benefit from the intervention.

The goal in infants and young children with CF, as opposed to older patients with more established lung disease, should focus on prevention of disease progression rather than treatment of existing disease. Rosenfeld et al have demonstratedthat testing therapeutic agents in infants and young children may require different endpoints capable of assessing progression of disease. However, to prove efficacy, these endpoints will require more than statistical significance; they must be associated with clinically meaningful responses. To make the next significant increase in survival, the CF community will again have to rise to the occasion and meet the current challenges in evaluating promising therapies. The youngest patients are depending on it.

\section{References}

1. Dasenbrook EC. Cystic fibrosis and survival in patients with advanced lung disease. BMJ. 2011; 342:d726. [PubMed: 21382942]

2. Cystic Fibrosis Foundation Patient Registry 2010 Annual Data Report. Bethesda, MD: 2011.

3. Davis PB, Drumm M, Konstan MW. Cystic fibrosis. Am J Respir Crit Care Med. 1996; 154:1229_ 1256. [PubMed: 8912731]

4. Ramsey BW, Davies J, McElvaney NG, et al. A CFTR potentiator in patients with cystic fibrosis and the G551D mutation. N Engl J Med. 2011; 365:1663-1672. [PubMed: 22047557]

5. Elkins MR, Robinson M, Rose BR, et al. A controlled trial of long-term inhaled hypertonic saline in patients with cystic fibrosis. N Engl J Med. 2006; 354:229-240. [PubMed: 16421364] 
6. Wark P, McDonald VM. Nebulised hypertonic saline for cystic fibrosis. Cochrane Database Syst Rev. 2009 CD001506.

7. Rosenfeld M, Ratjen F, Brumback L, et al. Efficacy and safety of inhaled hypertonic saline in infants and young children with cystic fibrosis: The ISIS randomized trial. JAMA. 2012

8. Vandevanter DR, Konstan MW. Outcome measures for clinical trials assessing treatment of cystic fibrosis lung disease. Clinical Investigation. 2012; 2:163-175.

9. Davis SD, Brody AS, Emond MJ, Brumback LC, Rosenfeld M. Endpoints for clinical trials in young children with cystic fibrosis. Proc Am Thorac Soc. 2007; 4:418-430. [PubMed: 17652509]

10. Davis SD, Rosenfeld M, Kerby GS, et al. Multicenter evaluation of infant lung function tests as cystic fibrosis clinical trial endpoints. Am J Respir Crit Care Med. 2010; 182:1387-1397. [PubMed: 20622043]

11. Mott LS, Park J, Murray CP, et al. Progression of early structural lung disease in young children with cystic fibrosis assessed using CT. Thorax. 2011 EPUB Ahead of Print.

12. Altes TA, Eichinger M, Puderbach M. Magnetic resonance imaging of the lung in cystic fibrosis. Proc Am Thorac Soc. 2007; 4:321-327. [PubMed: 17652494]

13. Amin R, Subbarao P, Jabar A, et al. Hypertonic saline improves the LCI in paediatric patients with CF with normal lung function. Thorax. 2010; 65:379-383. [PubMed: 20435858]

14. Belessis Y, Dixon B, Hawkins G, et al. Early cystic fibrosis lung disease detected by bronchoalveolar lavage and lung clearance index. Am J Respir Crit Care Med. 2012; 185:862-873. [PubMed: 22323305]

15. Ramsey BW, Banks-Schlegel S, Accurso FJ, et al. Future Directions in Early Cystic Fibrosis Lung Disease Research: An NHLBI Workshop Report. Am J Respir Crit Care Med. 2012; 185:887-892. [PubMed: 22312017]

16. Flume PA, O'Sullivan BP, Robinson KA, et al. Cystic fibrosis pulmonary guidelines: chronic medications for maintenance of lung health. Am J Respir Crit Care Med. 2007; 176:957-969. [PubMed: 17761616] 